\title{
Coanda effect in valves
}

\author{
Václav Uruba ${ }^{1,2,{ }^{*}}$, Pavel Procházka ${ }^{1}$, Vladislav Skála $^{1}$ \\ ${ }^{1}$ Institute of Thermomechanics, AS CR, v. v. i., Dolejškova 5, Praha 8, Czechia \\ ${ }^{2}$ University of West Bohemia, Faculty of Mechanical Engineering, Universitní 22, Plzeň, Czechia
}

\begin{abstract}
Coanda effect takes place in flow within valves diffuser for certain conditions. The valve plug in half-closed position forms wall-jet, which could be stable or instable, depending on geometry and other conditions. This phenomenon was subject of experimental study using time-resolved PIV technique. For the acquired data analysis the special spatio-temporal methods have been used.
\end{abstract}

\section{Introduction}

Recently the Coanda principle has become increasingly used in a wide variety of applications in many fields of applied dynamics of fluids - see e.g. [2]. In addition, this effect could be observed in the natural world. Devices containing a jet flow employing this principle usually offer substantial flow deflection, and enhanced turbulence levels and entrainment compared with a free jet flow. However, some disadvantages are connected with this phenomenon as jet flow detachment, considerable local increase of turbulence level, and appearance of quasi-periodical structures in the flowfield resulting in generation of pressure fluctuations associated with increased noise level. Physical mechanisms connected with those phenomena has not been explored in details yet.

The presented paper is contribution to study of the Coanda effect in some special cases which could occur in valves.

\section{Coanda effect}

The Coanda effect is a phenomenon that was first observed in 1910 by Romanian mathematician and engineer Henri Coanda. He discovered that when air was ejected from a rectangular nozzle, it would attach itself to an inclined flat plate connected to the nozzle exit. Emphasizing the need for a sharp angle between the nozzle and the flat plate, Coanda then applied the principle to a series of deflecting surfaces, each at a sharp angle to the previous one, and succeeded in turning flows through angles as large as 180 degrees. He stated that "when a jet of fluid is passed over a curved surface, it bends to follow the surface, entraining large amounts of air as it does so", and this phenomenon has become known as the "Coanda Effect".

Ability of the jet flow to follow the curved surface is determined by relevant conditions. The most important among them is the jet flow structure, and, of course, the surface shape which is to be followed. As a result, the jet flow follows the curved surface (or not) and the topology and dynamics of the emerging flow-field defined by the wall-jet flow. The same condition determine the structure of the flow-field and possible presence of instabilities.

The Coanda effect produces the attached boundary layer on a curved surface, however its ability has limits. For certain conditions the Coanda effect stops working and the boundary layer detaches. This process is of stability nature and could occur suddenly and random or quasi-periodical in time.

\section{Experimental setup}

The valve in question is depicted schematically in Fig. 1. It is a typical valve with side inlet used e.g. in a steam turbines. All parts of the valve are of circular crosssection. The diffuser angle is 7 degrees.

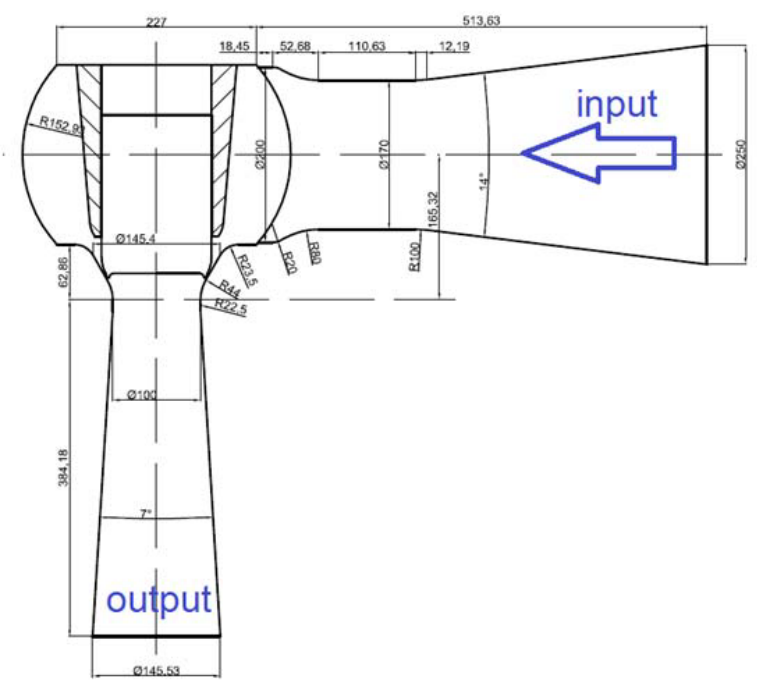

Fig. 1. The valve schema.

* Corresponding author: uruba@it.cas.cz 
For the purpose of experimental research, the simplified model of rectangular cross-section and only right-hand valve half was designed - see Fig. 2

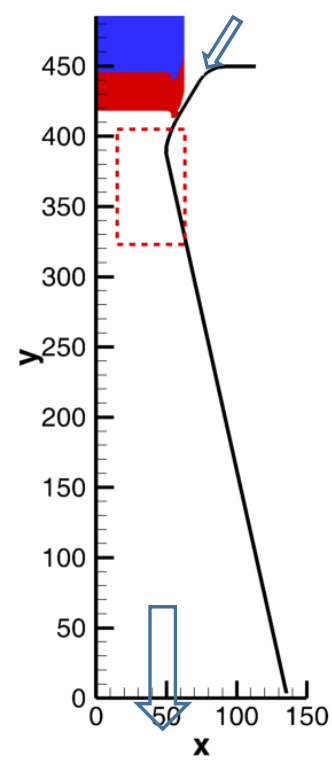

Fig. 2. The valve model.

In Fig. 2 the valve plug is shown in the two extremal positions, fully closed (in red) fully open (in blue). Position of the plug is described by the shift value $h$ in $\mathrm{y}$ direction with origin in fully closed position. So, the fully closed position corresponds to $h=0 \mathrm{~mm}$ and fully open $h=30 \mathrm{~mm}$. The shift $h$ determines the jet width, in fact. The arrows indicate flow directions and dashed red rectangle the measuring area. The angle of the halfdiffuser of the model was chosen 12.5 degrees.

The existing blow-down facility of Institute of Thermomechanics, Prague, has been used for experiments. The model was located on the output from the contraction nozzle of a cross-section of $250 \times 100 \mathrm{~mm}^{2}$. Velocity of air approx. $10 \mathrm{~m} / \mathrm{s}$ was used for most experiments.

\section{Methods and Procedures}

Time-resolved PIV measuring technique has been applied. The single camera method provided instantaneous in-plane velocity components in the plane of symmetry of the valve parallel to the mean flow. Frequency of evaluated velocity fields was $2 \mathrm{kHz}$ (i.e. 2000 measurements per second). The records consist of 4000 measurements, covering $2 \mathrm{~s}$ of time evolution. For seeding particles, a standard SAFEX fog generator has been used.

The instantaneous velocity fields have been evaluated from double-snaps using Dantec Dynamics A/S PIV software DynamicStudio version 3.40. The standard 3-step adaptive correlation method with consequent reduction of interrogation area has been used. The final size of interrogation area was $32 \times 32$ pixels with $50 \%$ overlap. This method considerably reduces random error of resulting velocities, it can be estimated within $1.5 \%$ of maximum velocity (see [1]). However, all methods used for the data analysis are of statistical nature, therefore random errors of evaluated modes are negligible. Possible systematic error is connected with used tracing particles with typical size of $1 \mu \mathrm{m}$. For the questioned case, the systematic error is within $1 \%$ of the maximum velocity.

The time-mean statistics have been evaluated. For the dynamical behaviour the Oscillation Pattern Decomposition (OPD) method has been adopted. The OPD method allows for extraction of quasi-periodical modes. The OPD method is described in details in [3].

Each OPD mode is characterised by complex topology and frequency $f$, e-folding time $\tau$ and periodicity $p$. Frequency of quasi-periodical mode evolution, e-folding time is mean time to the mode amplitude reduction by factor $\mathrm{e}$, it could be understood as reciprocal value of the mode damping. The periodicity evaluates the e-folding time in multiples of the process periods. If the $p$ is greater than 0.343 the mode is considered to be oscillating, otherwise it is nonoscillating or decaying in nature (see [5]).

\section{Results}

The results are to be shown evaluated within the rectangular area - see Fig. 2. The plug shift $h$ was changed in broad range: $2.5,5,7.5,10,12.5,15,17.5,20$ and $30 \mathrm{~mm}$ respectively. For presentation only selected results have been chosen. However only the case $h=30 \mathrm{~mm}$ shows failure of the Coanda effect with fully detached boundary layer.

\subsection{Time mean results}

For the presentation of time-mean characteristics the two typical cases have been chosen: the case of $h=10 \mathrm{~mm}$, when the flow is attached to the curved wall and the Coanda effect is working and $h=30 \mathrm{~mm}$, detached flow and Coanda effect failure. The first case will be referred hereinafter as (a), the latter as (b).

In Fig. 3 there are time-mean vector-fields with vector-lines added in arbitrary manner (blue colour) for the cases (a) and (b).

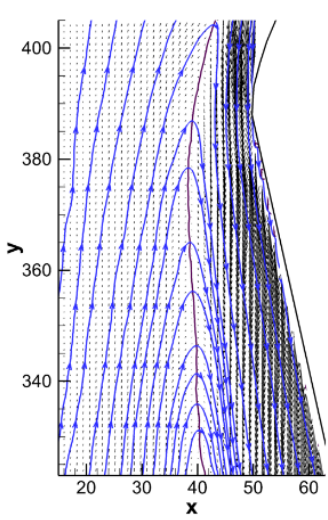

(a)

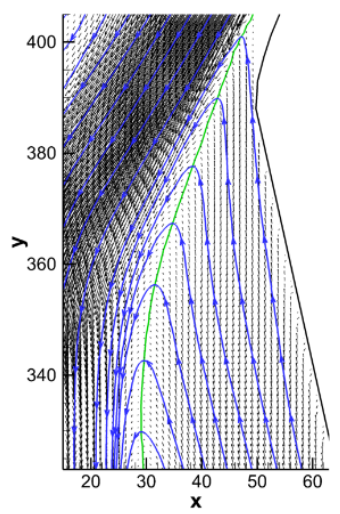

(b) 
Fig. 3. Vector-lines of time-mean velocity vector field, (a) $h=10 \mathrm{~mm}$, (b) $h=30 \mathrm{~mm}$.

The brown and green lines represent boundary of area with streamwise (down) and back (up) flow, corresponding to the vanishing streamwise velocity component. For the case (a) the back-flow is located in the left-hand part, while for the case (b) is back-flow in the right. Please note that even if the back-flow region is relatively big in area extend, the backward oriented velocity moduli are relatively very small and thus the backward oriented flow-rate is also small (compare with Fig. 5, where moduli distributions are shown).

The back-flow boundary position is function of the valve plug shift, this is demonstrated in Fig. 4, were boundaries for all examined shifts are shown. The $h$ value is put close to relevant curve.

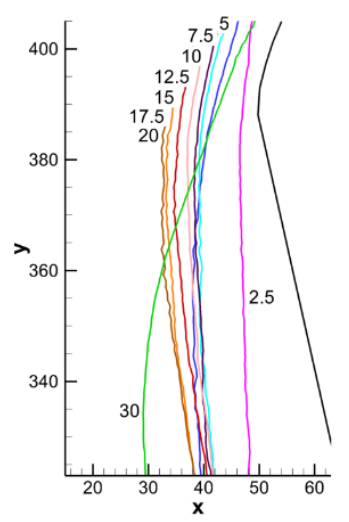

Fig. 4. Zero streamwise velocity component lines, $h$ is variable.

The position of the zero streamwise velocity component depends systematically on the plug shift (i.e. width of the jet). This is evident in the upper part $(y=390 \mathrm{~mm})$ : the bigger shift $h$ and width of the jet, the thicker the area of attached flow in the left. However more-or-less the same attached area thickness is established very quickly, at the lower area border $(y=330 \mathrm{~mm})$. Only the case of $h=2.5 \mathrm{~mm}$ shows considerably thinner attached area. This effect could be attributed to viscose effects, when the gap between plug and valve wall is very thin.

The case $h=30 \mathrm{~mm}$ (green line) represents an exception, of course, as the flow is detached.

In Fig. 5 there are mean velocity moduli distributions for (a) and (b) cases respectively. Much higher velocities are indicated in streamwise direction flow regions than in the backward flow.

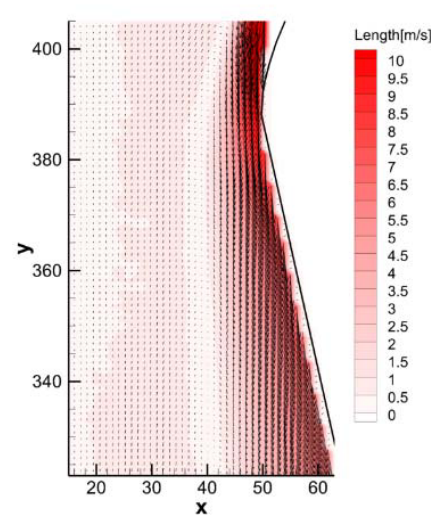

(a)

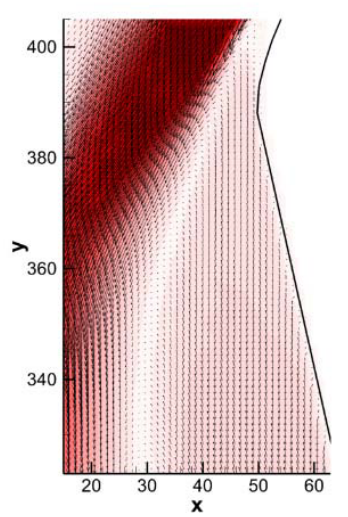

(b)
Fig. 5. Time-mean velocity vector fields and moduli distributions, (a) $h=10 \mathrm{~mm}$, (b) $h=30 \mathrm{~mm}$

To indicate the intensity of dynamical activity, the variances of velocity components are evaluated. The variances of both evaluated velocity components in a given point were added, distributions of sum of variances are shown in Fig. 6.

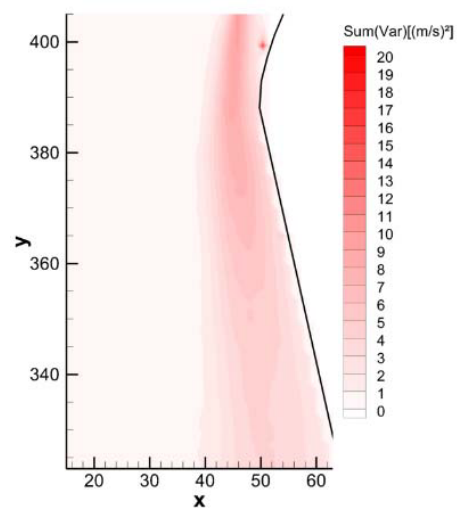

(a)

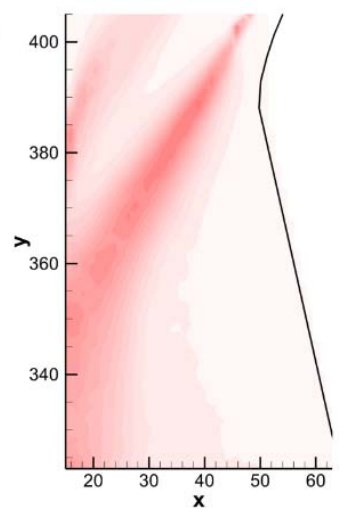

(b)
Fig. 6. Distributions of sum of velocity components variances, (a) $h=10 \mathrm{~mm}$, (b) $h=30 \mathrm{~mm}$

The distributions of velocity variance indicate high fluctuating activity within whole volume of the wall-jet in the case (a), while for the case (b) maximum activity is located to the lower border of the free jet, less intensive local maximum within the upper jet border. The same conclusion could be made from the correlation coefficient distributions in Fig. 7. Correlation coefficient indicates locations with high production of turbulence. 


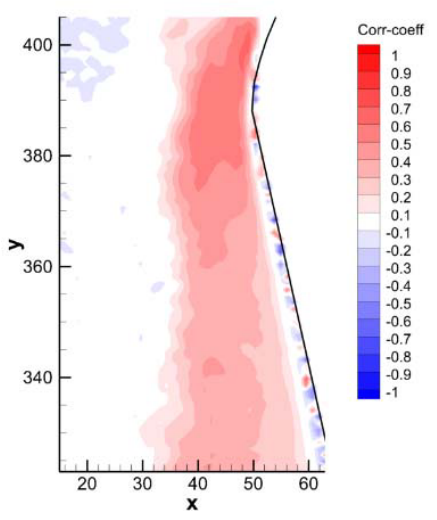

(a)

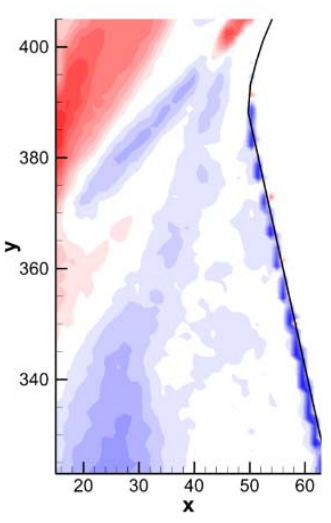

(b)
Fig. 7. Correlation coefficient distributions, (a) $h=10 \mathrm{~mm}$, (b) $h=30 \mathrm{~mm}$

The information connected with flow dynamics is however very limited. More detailed information will be provided in the following paragraph.

\subsection{Dynamics}

The detailed study of dynamics of flow-field has been performed using the OPD method. The mode with strong oscillating capacity are to be presented here, see the Tab. 1.

Table 1. Results of OPD analysis.

\begin{tabular}{|c|c|c|c|c|}
\hline$h[\mathrm{~mm}]$ & OPD No. & $f[\mathrm{~Hz}]$ & $\tau[\mathrm{ms}]$ & $p[1]$ \\
\hline 5 & 2 & 34.8 & 17.30 & 0.601 \\
\hline 5 & 4 & 82.3 & 7.94 & 0.653 \\
\hline 5 & 7 & 187.9 & 3.174 & 0.596 \\
\hline 10 & 4 & 67.5 & 7.94 & 0.535 \\
\hline 10 & 5 & 146.9 & 5.94 & 0.872 \\
\hline 10 & 11 & 129.4 & 2.48 & 0.533 \\
\hline 15 & 8 & 123.1 & 5.14 & 0.633 \\
\hline 15 & 10 & 198.5 & 3.74 & 0.742 \\
\hline 15 & 12 & 231.9 & 3.40 & 0.788 \\
\hline 20 & 3 & 101.7 & 7.03 & 0.714 \\
\hline 20 & 7 & 211.1 & 5.41 & 1.143 \\
\hline 20 & 8 & 152.8 & 4.55 & 0.695 \\
\hline 30 & 4 & 221.1 & 7.57 & 1.674 \\
\hline 30 & 6 & 126.2 & 6.28 & 0.793 \\
\hline 30 & 9 & 331.6 & 5.64 & 1.870 \\
\hline
\end{tabular}

In Table 1 all relevant parameters of selected OPD modes are shown. For each $h$ position 5, 10, 15, 20 and $30 \mathrm{~mm}$ respectively, the mode frequency $f$, e-folding time $\tau$ and periodicity $p$ is given for the 3 selected OPD modes.

The OPD topology will be presented in form of real and imaginary parts, velocity vector field and corresponding vorticity field (red - positive, blue negative). As an example the OPD mode 2 for $h=5 \mathrm{~mm}$ is depicted in Fig. 8.
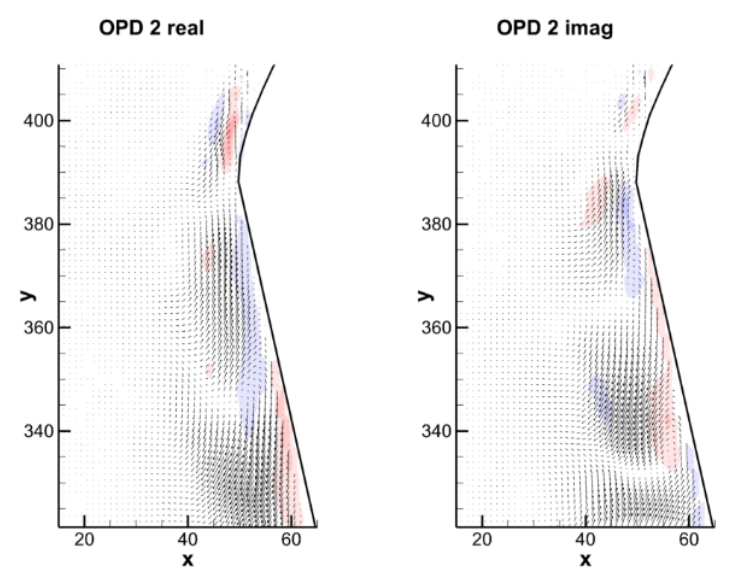

Fig. 8. OPD mode 2 for $h=5 \mathrm{~mm}$, real and imaginary parts.

The oscillating mode in Fig. 8 is a typical traveling mode, the vortical structures travel in space with a small modification within the oscillation period. The real and imaginary parts of the mode shows very similar topology, the main difference is in positions of vortical structures.

All other modes are of very similar nature, so we will present only real parts of OPD modes, the imaginary parts are similar, only shifted in space.

In Fig. 9 there are OPD modes 4 and 7 for $h=5 \mathrm{~mm}$.
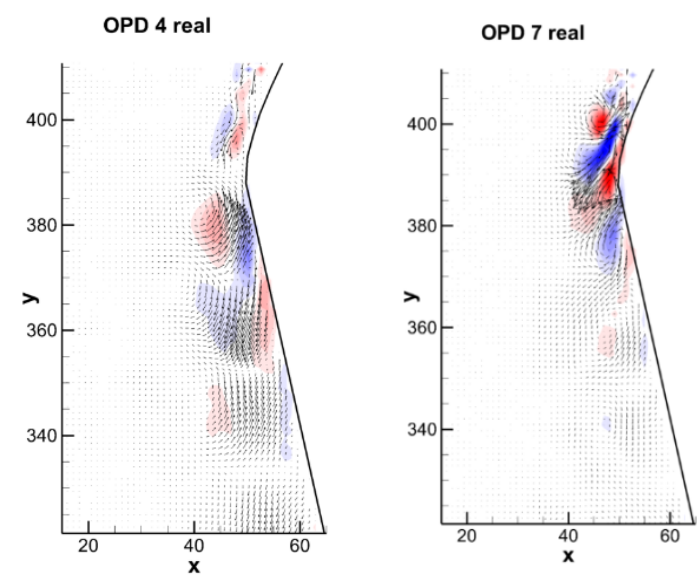

Fig. 9. OPD modes 4 and 7 for $h=5 \mathrm{~mm}$, real parts.

The traveling vortices could be recognized within the wall jet (mode OPD 4), in the case of OPD 7 very intense vortical structures are located just in the region of the valve seat. 

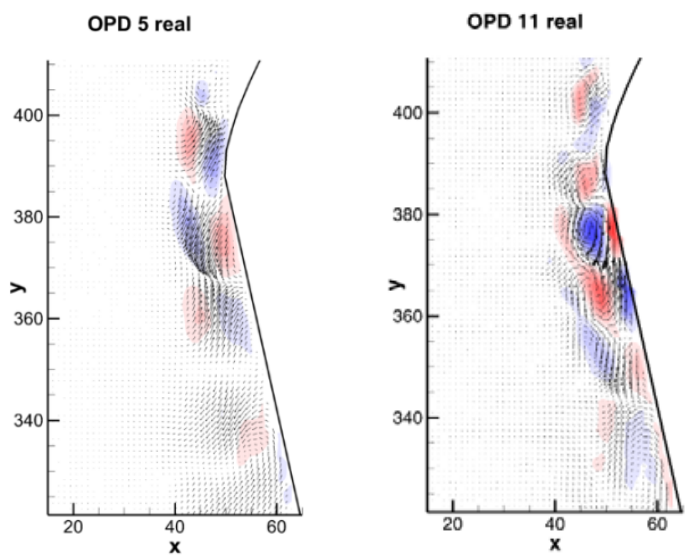

Fig. 10. OPD modes 5 and 11 for $h=10 \mathrm{~mm}$, real parts.

The OPD modes for $h=10 \mathrm{~mm}$ in Fig. 10 show the vortical structures shifted downstream in the streamwise direction. Further downstream shift could be visible for even bigger shifts $h=15 \mathrm{~mm}$ and $20 \mathrm{~mm}$ in Figs. 11 and 12. The dynamical structures became weaker.
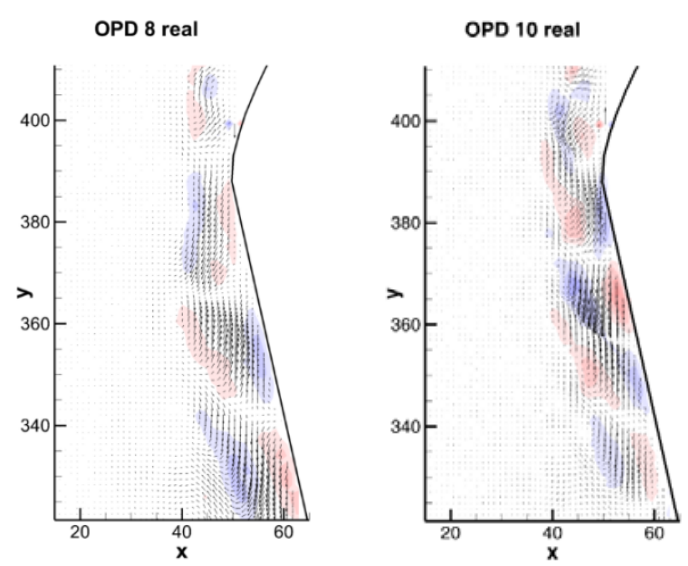

Fig. 11. OPD modes 8 and 10 for $h=15 \mathrm{~mm}$, real parts.
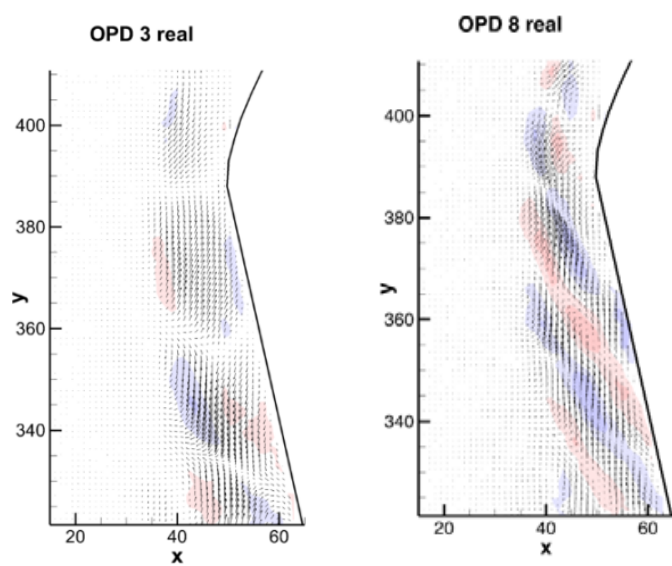

Fig. 12. OPD modes 3 and 8 for $h=20 \mathrm{~mm}$, real parts.

The last plug position $h=30 \mathrm{~mm}$ exhibits failure of Coanda effect and detachment of the jet. The dynamics is completely different. The travelling dynamical structures are moving together with the jet, staying attached to it.
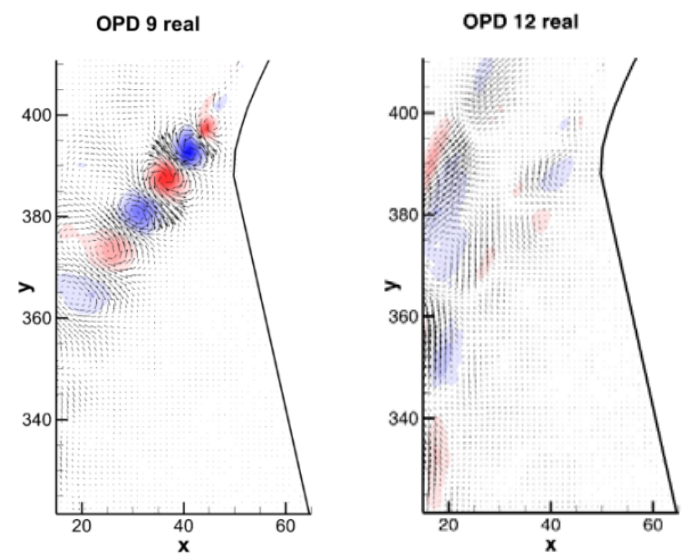

Fig. 13. OPD modes 9 and 12 for $h=30 \mathrm{~mm}$, real parts.

In Fig. 13 the OPD mode 9 shows very distinct and well defined vortex train in the region of the free jet lower border. The mode OPD 12 represents dynamics of whole free jet.

\section{Conclusions}

The mean flow characteristics and dynamical modes of the flow-field in the valve diffuser were shown. Coanda effect is active for narrow wall jets, i.e. small valve plug shifts. The flow-field dynamics is typically limited to the wall-jet region. With growing wall-jet width the dynamical vortical structures shift further downstream and became weaker. For big shifts of the valve plug the Coanda effect fails and the jet detaches from the wall. The resulting free jet exhibits very intense dynamics. On the lower border of the jet-flow the distinct vortex street with alternative orientation was detected.

\section{Acknowledgement}

The authors gratefully acknowledge the support of the Technology Agency of the Czech Republic by means of projects TA04020129 and TA04011437.

\section{References}

1. DynamicStudio User's Guide, 2015

2. C. Lubert, On Some recent applications of the Coanda effect, The Journal of the Acoustical Society of America 128 (4) :2286, October 2010

3. V. Uruba, Decomposition methods in turbulent research, EFM11, EPJ Web of Conferences 25 01095 (2012)

4. V. Uruba, O. Hladík, P. Jonáš, Dynamics of secondary vortices in turbulent channel flow, ETC13, Journal of Physics Conference Series 318 062021 (2011)

5. V. Uruba, Near wake dynamics around a vibrating airfoil by means of PIV and Oscillation Pattern Decomposition at Reynolds number of 65000 , Journal of Fluids and Structures 55 pp. 372-383 (2015) 